\title{
La direction de recherche phénoménologique en psychopathologie
}

Karl Jaspers

Traducteur : Simon Calenge

URL : http://journals.openedition.org/alter/1454

DOI : $10.4000 /$ alter. 1454

ISSN : 2558-7927

Éditeur :

Association ALTER, Archives Husserl (CNRS-UMR 8547)

\section{Édition imprimée}

Date de publication : 1 octobre 2011

Pagination : 229-246

ISBN : 978-2-9522374-7-5

ISSN : $1249-8947$

\section{Référence électronique}

Karl Jaspers, «La direction de recherche phénoménologique en psychopathologie », Alter [En ligne] 19 | 2011, mis en ligne le 01 janvier 2020, consulté le 23 janvier 2020. URL : http:// journals.openedition.org/alter/1454; DOI : 10.4000/alter.1454 


\title{
LA DIRECTION DE RECHERCHE PHÉNOMÉNOLOGIQUE EN PSYCHOPATHOLOGIE ${ }^{1}$
}

\author{
Karl Jaspers
}

[314] En examinant les malades mentaux, on a l'habitude de distinguer symptômes objectifs et symptômes subjectifs. Les symptômes objectifs sont tous les processus dont la manifestation est perceptible aux sens : les réflexes, les mouvements et le visage susceptibles d'être enregistrés et photographiés, les excitations motrices, les extériorisations langagières, les productions écrites, les actions, le mode de vie, etc. ; appartiennent en outre aux symptômes objectifs toutes les opérations mesurables comme la capacité de travail, l'exercice, les opérations mnésiques, etc. On compte enfin habituellement parmi ces symptômes les idées délirantes, les déformations de souvenirs et d'autres symptômes semblables, en un mot les contenus rationnels des productions langagières que nous ne pouvons que comprendre et non percevoir par les sens, et que nous comprenons rationnellement,

\footnotetext{
1. Initialement paru en 1912 dans la Zeitschrift für die gesammte Neurologie und Psychiatrie, tome 9, p. 391-408, cet article a été republié dans les Gesammelte Schriften zur Psychopathologie, Berlin / Göttingen / Heidelberg, Springer, 1963, p. 314 à 328, dont la pagination est reproduite entre crochets dans le corps du texte. Cet article est le second d'une série de trois qui constitue un des chantiers où s'élabore la première version de la Psychopathologie générale et parmi lesquels on compte L'analyse des perceptions trompeuses (1911) et Configurations causales et compréhensibles entre destin et psychose dans la Dementia praecox (schizophrénie) (1913). Dans ces écrits, Jaspers, alors jeune psychiatre à la clinique de Heidelberg, s'engage dans une réflexion relative aux maladies mentales en s'efforçant de libérer l'espace nécessaire à une méthode compréhensive dans laquelle la phénoménologie de Husserl joue un rôle décisif. L'analyse des perceptions trompeuses tente de donner une description complète des phénomènes normaux et pathologiques voisins de la perception en recourant à la distinction husserlienne entre acte et contenu. Jaspers s'appuie sur les Recherches logiques, et l'ensemble de son travail sur la phénoménologie restera attaché à cette première phénoménologie descriptive antérieure à la réduction transcendantale. En 1912, La direction de recherche réfléchit sur ce premier apport théorique et sur cette distinction entre actes et contenus en intégrant la phénoménologie à la psychopathologie. La phénoménologie devient alors plutôt une méthode qu'une conception de la structure du psychique : l'étape préparatoire de la psychologie compréhensive que Jaspers appelle de ses vœux (N.d.T.).
} 
c'est-à-dire sans l'aide de quelque transposition interne dans l'âme par la pensée.

Alors que tous les symptômes objectifs peuvent être montrés immédiatement et présentés de façon convaincante dans leur réalité à tous ceux qui ont des sens et une pensée logique, les symptômes subjectifs renvoient à quelque chose que l'on nomme justement subjectif par opposition à la perception sensible et à la pensée logique : ils ne peuvent être vus par les organes des sens, mais peuvent seulement être saisis par la transposition dans l'âme d'autrui, par empathie, ils peuvent seulement être co-vécus et non devenir l'objet d'une intuition interne par la pensée. Les symptômes subjectifs sont tous les mouvements de l'âme et tous les processus internes que nous pensons saisir immédiatement dans les manifestations sensibles, comme l'angoisse, la tristesse, la gaieté, et qui accèdent ainsi à une «expression». Les symptômes subjectifs sont en outre tous les vécus et phénomènes psychiques décrits par les malades et qui ne nous sont accessibles que médiatement par leur jugement et leur représentation. Ce sont enfin les processus psychiques interprétés et rendus accessibles à partir des bribes (actions, modes de vie, etc.) des deux genres de données précédentes.

Une opposition de valeur est habituellement liée à la distinction entre symptômes objectifs et subjectifs. Les symptômes objectifs sont les seuls qui soient sûrs - on ne peut entreprendre de recherche scientifique qu'à leur propos. Quant aux symptômes subjectifs, ils sont certes indispensables a toute orientation provisoire, mais leur établissement est tenu pour très incertain et stérile au regard d'examens scientifiques plus poussés. Certains chercheurs exigent de fonder la connaissance des maladies mentales sur les seuls symptômes objectifs, [315] l'idéal étant d'éliminer complètement tous les symptômes subjectifs. C'est une conception qui a ses représentants plus ou moins conséquents aussi bien en psychologie qu'en psychiatrie. Une psychologie objective s'oppose à une psychologie subjective. La première ne veut travailler qu'avec des donnees objectives et conduit à une psychologie sans âme; la seconde, qui ne méconnaît du reste jamais la valeur de la première, s'en tient à l'auto-observation, à l'analyse subjective, au constat des modes d'être du psychique, de la particularité des phénomènes, et $\mathrm{n}^{\prime}$ accorde de valeur à de tels examens que s'ils sont menés sans aucun point d'appui objectif. Les exemples de la doctrine de la perception sensible, des mesures de la mémoire et de l'examen de la courbe de travail avec ses composantes, forment de larges chapitres de la psychologie objective. Par ce dernier exemple - l'examen de la courbe de travail - nous prenons conscience que ces examens mènent à une élimination méthodique du psychique. On n'examine pas les sentiments d'épuisement (la fatigue), mais l'epuisement objectif. Tous les concepts tels que la fatigabilité, les capacités de rétablisse- 
ment, d'endurance, de résistance, l'effet des pauses, etc., se rapportent à des opérations objectivement mesurables alors qu'il est tout à fait indifférent de savoir s'il s'agit dans ces opérations d'une machine, c'est-à-dire d'un organisme vivant sans âme, ou d'un homme psychique. De tels examens objectifs recourent toutefois secondairement - naturellement à juste titre - à l'interprétation de symptômes subjectifs ou à leur comparaison avec les opérations objectives. On recourt alors à la psychologie subjective dont il est question dans cet essai. La psychologie objective obtient indubitablement des résultats plus palpables que la psychologie subjective, plus sûrs, plus facilement saisissables pour tous. Cependant la différence n'est que graduelle en ce qui concerne le niveau de certitude, alors qu'elle est principielle au regard du mode de certitude. La psychologie subjective conduit toujours au remplissement absolu de ses concepts et de ses visées dans les présentifications internes et l'intuition psychique, alors que la psychologie objective trouve son ultime accomplissement dans les perceptions sensibles incontestables, dans des chiffres, des courbes ou des contenus rationnels.

Que veut la psychologie subjective tant décriée? Tandis que l'objective devient partiellement ou entièrement physiologie par la mise hors circuit du psychique, la subjective veut justement faire de la vie psychique un objet. Plus généralement, elle se demande de quoi dépend la vie psychique, ce que sont ses effets, quelles sont les configurations qu'il est possible d'y appréhender. Sa visée authentique est de répondre à de telles questions. Mais à chaque question, elle se voit placée devant la nécessité de se préciser à elle-même et aux autres la nature du vécu psychique déterminé qu'elle vise exactement. Elle se trouve en effet face à une multiplicité de phénomènes psychiques qu'elle ne peut considérer dans leur globalité ; elle ne peut examiner ces phénomènes en général, mais seulement les considérer dans leur singularité. Avant donc qu'elle ne commence son propre travail, elle doit se présentifier et clarifier les phénomènes psychiques qu'elle vise, les distinguer de ceux avec lesquels ils ne doivent pas être confondus et de ceux qui leur ressemblent, etc. Lorsque le travail préalable de présentification, de délimitation clarifiante et de mise en ordre des phénomènes psychiques [316] est mené de manière autonome, alors naît la phénoménologie. C'est parce que ce travail est difficile et vaste qu'il doit être provisoirement mené seulement pour lui-même. Aussi longtemps qu'on ne s'est pas saisi de cet examen autonome et méthodique, le travail phénoménologique préalable reste borné à des réflexions justifiées ad hoc sans lien entre elles, dépendant de l'inspiration du moment, et dans lesquelles on peut certes découvrir de bons commencements, mais où la recherche ne peut demeurer.

Au sein de la recherche psychologique, c'est Husserl qui a fait le 
pas décisif vers une phénoménologie méthodique, préparé en cela par Brentano et son école et par Theodor Lipps. Pour la psychopathologie, il existe toute une série de commencements phénoménologiques ${ }^{2}$, mais elle n'est pas devenue une direction de recherche généralement reconnue, accomplissant le travail préalable aux tâches propres de la psychopathologie. Comme il y a ici beaucoup de tâches auxquelles chacun peut travailler, une présentation programmatique des buts et des méthodes nous semble opportune.

Dans la vie quotidienne, personne ne pense à ses propres phénomènes psychiques isolés ou à ceux des autres. Nous sommes intérieurement toujours dirigés sur ce que visent nos vécus, et non sur les processus de notre âme au sein du vécu. Nous comprenons les autres, non par une observation ni par une analyse de leur vie psychique, mais en vivant avec eux dans l'ensemble des événements, des destins et des actions. Et lorsque nous observons effectivement les vécus psychiques eux-mêmes, nous ne le faisons habituellement que dans la configuration formée par les motifs et les conséquences que nous comprenons, ou bien nous classons les personnalités dans des catégories caractérologiques. Nous n'avons jamais l'occasion d'observer et de décrire les phénomènes psychiques isolés - une perception pour soi, un sentiment - dans leur manifestation, dans leur mode d'être, dans leur donation. C'est justement ainsi que le psychiatre se conduit à l'égard du malade. Il peut avoir un vécu commun avec le malade dans la mesure où un tel vécu se produit sans qu'une réflexion préalable soit requise, et il peut parvenir à une compréhension tout a fait personnelle, informulable et incommunicable, qui reste cependant pour lui un pur vécu et ne devient jamais connaissance consciente. Il acquiert certes de l'expérience dans la compréhension, mais pas une collection d'expériences conscientes susceptibles de permettre des comparaisons - plus claires que lorsqu' on a seulement affaire à de vagues impressions et à des «sentiments »-, d'être ordonnées, établies et mises à l'épreuve.

Extraordinairement satisfaisante pour un individu singulier, cette attitude de la compréhension purement empathique (miterlebenden), qui, selon la disposition de chacun, peut constituer la fin dernière de toute une vocation, est toutefois subjective en un sens particulier. Et lorsqu'on expose de telles affirmations singulières, qu'on produit des

\footnotetext{
2. Le livre de Kandinsky, Kritische und klinische Betrachtungen im Gebiete der Sinnestaüchungen, Berlin, 1885, est presque entièrement phénoménologique, abstraction faite des remarques théoriques à négliger et qui ne nuisent pas à l'ouvrage. Oesterreich, Die phänomenologie des Ich in ihren Grundproblemen, Leipzig 1910, et Haecker, "Systematische Traumbeobachtungen », Archiv für Psychologie 21, 1, 1911, pratiquent méthodiquement une phénoménologie de tels phénomènes, qui sont particulièrement importants pour la psychopathologie. Dans deux études (Zur Analyse der Trugwahrnehmung, p. 191, et Die Trugwahrnehmungen, p. 252), j'ai moi-même contribué à ce travail.
} 
formulations à partir d'une telle compréhension d'ensemble, sans réflexion plus poussée, sans concepts fixes réguliers, [317] il faut caractériser ce genre de formulations de «purement » subjectives en un sens péjoratif. Elles ne sont en effet ni discutables ni vérifiables. Si nous pouvons encore tenir en très haute estime cet acte de compréhension a cause des précieux dons humains qui s'y montrent, nous ne pouvons cependant pas l'appeler science - qu'il s'agisse de l'acte de compréhension dans sa forme sublimée tel qu'il est pratiqué parmi les hommes de cercles cultivés depuis des siècles, ou de l'immersion empathique sans concept du psychiatre.

$\mathrm{Si}$, face à cela, une science psychologique veut encore se développer, elle doit d'abord prendre conscience que ce n'est qu'à titre d'idéal qu'elle recherche la claire compréhension du psychique, devenue pleinement consciente et représentable dans des formes fixes; elle cherche cette compréhension à laquelle les hommes les plus doués $\mathrm{n}^{\prime}$ accèdent en fait qu'inconsciemment, vaguement, personnellement et subjectivement ; elle doit prendre conscience qu'elle ne correspond même pas approximativement à cet idéal, mais qu'elle est bloquée dès ses commencements, qui, certes, ouvrent des perspectives. Cependant, dès ces commencements, un tel idéal est rejeté dans l'infini. Si beaucoup exercent leur compréhension personnelle pour leur satisfaction propre, tous sourient donc des tentatives de fixation psychologique consciente de concepts, les comparant à d'inoffensives platitudes, à des trivialités, dès lors qu'ils se placent du point de vue $\mathrm{d}^{\prime}$ une vision englobante, quoique vague ${ }^{3}$. Ce qui donne une valeur du point de vue scientifique, et seulement de ce point de vue, à de telles fixations psychologiques conscientes, c'est le fait qu'un savoir se joue en elles.

Cette attitude qui ne veut pas demeurer dans le vécu compréhensif, mais parvenir à un savoir communicable, vérifiable, discutable, est confrontée à une infinité de phénomènes psychiques extrêmement divers, où règnent encore des configurations peu claires et dont les liens et les effets restent à découvrir. Ici, le premier pas vers une saisie scientifique est sans aucun doute un démêler, un délimiter, un distinguer et un décrire de phénomènes psychiques déterminés, qui sont ainsi clairement présentifiés et correctement nommés par une expression déterminée. La présentification de ce qui arrive réellement au malade, de ce qu'il vit vraiment, de la manière dont quelque chose est donné à sa conscience, dont il se sent, etc., c'est là le commencement dans lequel on doit d'abord faire abstraction des configurations, du vécu

\footnotetext{
3. On ne peut nier que de nombreux psychologues en restent à des trivialités. On ne peut pas moins nier qu'en lieu et place d'une phénoménologie méthodique fondée comme science nous avons parfois affaire à une pseudopsychologie; le contenu de ce comprendre personnel et vague, du point de vue de la communicabilité, est simplement exposé dans des expressions aussi vagues - quoique érudites - plutôt qu'en allemand correct.
} 
en tant que tout, et en premier lieu des pensées additionnelles, des pensées de principes, des représentations théoriques. Seul ce qui est effectivement conscient doit être présentifié, tout ce qui n'est pas effectivement donné dans la conscience n'est pas disponible. Nous devons laisser de côté toute théorie transmise, toute construction psychologique ou toutes les mythologies matérialistes relatives aux processus cérébraux: nous devons nous tourner purement vers ce que nous pouvons comprendre, saisir, distinguer et décrire en son existence effective. Comme nous l'apprend l'expérience, c'est là [318] une tâche très difficile. Cette authentique absence phénoménologique de préjugé n'est pas une possession originaire, mais une acquisition difficile qui résulte d'un long travail critique et de nombreux efforts, souvent vains au sein de ces constructions et de ces mythologies. De même que, enfants, nous dessinons les choses non telles que nous les voyons mais telles que nous les pensons, de même, en tant que psychologues et psychopathologues, nous passons par une étape où nous pensons de quelque manière le psychique pour nous-mêmes, pour ensuite seulement nous diriger vers une saisie, immédiate et dépourvue de préjugés, du psychique tel qu'il est. Et cette attitude phénoménologique est un effort toujours renouvelé et un bien à acquérir par une victoire toujours nouvelle sur les préjugés : c'est l'attitude phénoménologique.

Comment faisons-nous, lorsque nous isolons, caractérisons et fixons conceptuellement des phénomènes psychiques? Nous ne pouvons figurer les phénomènes psychiques, les poser devant les yeux comme un objet de perception sensible. Nous pouvons seulement multiplier les voies permettant de présentifier un phénomène déterminé, et appeler chacun à en faire autant. La genèse, les conditions et constellations qui déterminent l'apparition de ce phénomène, les configurations dans lesquelles il est habituellement présent, les contenus objectifs qu'il est susceptible d'avoir, les comparaisons intuitives et les symbolisations, une espèce suggestive $\mathrm{d}^{\prime}$ orientation à laquelle parviennent le mieux les artistes, l'exposition de phénomènes déjà connus auparavant et qui jouent ici un rôle à titre d'éléments de ce qui est présent..., tout cela doit conduire de l'extérieur vers le phénomène psychique véritablement visé. $\grave{A}$ travers toutes ces voies s'intensifie un appel à la présentification des phénomènes visés, qui s'adresse aux autres, puis, dans l'usage ultérieur de ce que nous avons établi, à nous-mêmes. Plus les voies de présentification sont nombreuses et spécifiques, plus sûre est la caractérisation et la détermination du phénomène visé. Cette présentification autonome de phénomènes psychologiques renvoyant toujours à des [réalités] extérieures est la condition de tout travail en général portant sur le psychologique.

De même que l'histologue décrit les éléments morphologiques particuliers, certes minutieusement, mais seulement de manière à ce 
que chacun puisse ensuite les voir lui-même plus facilement, et qu'il doit présupposer ou provoquer ce voir-soi-même chez ceux qui veulent réellement le comprendre, de même le phénoménologue peut indiquer de nombreux caractères, distinctions, confusions, pour décrire les données psychiques qualitatives singulières. Cependant, il doit compter sur le fait que les autres ne pensent pas simplement en commun avec le malade, mais que, dans leurs conversations avec lui, ainsi que dans leur propre présentification, ils voient en commun avec lui. Ce voir n'est pas sensible, mais c'est un voir compréhensif. Pour faire un seul pas en avant en phénoménologie, on doit exercer et avoir conçu cet authentique et irréductible fait ultime, ce «faire accéder à la donation », ce " comprendre », ce "saisir », cet « intuitionner », ce «se présentifier ». C'est seulement ainsi qu'il est possible de parvenir à une critique féconde visant aussi bien les constructions que les dénégations stériles qui font obstacle à toute possibilité de progrès. Qui n'a pas d'yeux pour voir, ne peut pas pratiquer I'histologie ; qui se défie ou n'est pas doué pour se présentifier le psychique et pour l'observer de façon vivante ne peut pas concevoir la phénoménologie.

[319] Cette ultime et irréductible qualité du phénomène psychique - de ne pouvoir être visé dans son identité que par cet appel a le voir à travers la multiplicité des voies qui y mènent - se trouve déjà dans les qualités sensibles les plus simples, par exemple le bleu, le rouge, la couleur, le son, tout comme dans l'intuition de l'espace, la conscience d'objet, la perception, la représentation, la pensée, etc. Nous l'avons dans le domaine de la psychopathologie, par exemple, dans la pseudo hallucination, dans le déjà-vu, dans l'aliénation du monde perceptif, dans l'expérience de son propre dédoublement, dans la dépersonnalisation, etc., toutefois tous ces noms ne désignent qu'un groupe de phénomènes psychiques qui diffèrent encore entre eux selon d'autres nuances.

Pour la présentification de toutes ces ultimes qualités phénoménologiques, nous avons déjà recouru à de multiples expressions comme voir, regarder, sentir, comprendre... Avec toutes ces expressions, le même [fait] ultime est compris, à savoir le vécu remplissant nos concepts, vécu qui est la même chose dans le domaine psychologique que la perception sensible dans le domaine des sciences de la nature. Tout comme les perceptions sensibles sont suscitées par la démonstration d'un objet, de même ces présentifications compréhensives et empathiques le sont par les voies qui viennent d'être nommées, par la saisie immédiate des phénomènes expressifs, par l'immersion dans l'auto-description. Il résulte de cette manière de s'exprimer que l'empathie et la compréhension ne sont pas un simple phénomene ultime, mais contiennent encore une série d'éléments à différencier. Tout comme la perception, cette empathie commence d'abord par 
assigner à la phénoménologie, dont elle est le fondement, la tâche de la recherche génétique 4 . Mais aucun de ces deux phénomènes ne nous intéresse ici. On se contentera de voir comment se réalise notre savoir dans les vécus d'empathie et de compréhension, et d'interroger la fiabilité de cette manière dont les faits nous sont accessibles. Si on a reconnu ces vécus, de manière analogue aux vécus perceptifs, comme quelque chose d'ultime, on doit pouvoir répondre à la question de leur fiabilité : les moyens techniques de conservation des intuitions à des fins de comparaison et les autres procédés du même type sont si imparfaits dans le domaine des vécus empathiques qu'on doit lutter avec beaucoup plus de difficultés que dans le domaine de la perception sensible; on établit ici leur fiabilité par la comparaison, la répétition, la vérification des vécus empathiques, par les présentifications, de même que, dans le sciences de la nature, les résultats découverts dans la perception sensible sont comparés, répétés et vérifiés. L'incertitude règne dans les deux domaines. Qu'elle soit plus grande du côté de la psychologie est incontestable. Mais ce n'est qu'une différence de degrés.

Cela revient à peu près au même de présentifier ses propres vécus psychiques passés ou de présentifier ceux des autres hommes. Il y a une différence plus significative entre les observations ${ }^{5}$ tirées de l'auto-observation méthodique expérimentale faite sur les vécus persévérants [320] et les simples présentifications compréhensives. Pour nos recherches sur les phénomènes psychopathologiques, seules ces dernières sont prises en considération, puisque les malades peuvent rarement être amenés à l'auto-observation au premier sens, et s'ils le peuvent, c'est seulement dans certaines circonstances liées à des pathologies simples (perceptions trompeuses dans la conscience réfléchie, agnosie etc.). Nous pouvons néanmoins espérer un progrès significatif de cette présentification compréhensive des phénomènes psychiques des malades mentaux grâce aux concepts qui sont dégagés par la recherche phénoménologique de la première espèce [la présentification de vécus propres].

Il y a trois moyens qui permettent d'analyser et d'établir phénoménologiquement ce que vit effectivement le malade : première-

\footnotetext{
4. La littérature générale sur l'empathie et le comprendre peut être facilement trouvée dans le rapport de Geiger sur le quatrième congrès de psychologie expérimentale, Über das Wesen und die Bedeutung der Einfühlung (1910). [Il s'agit ici non de la tâche d'une psycho-physique génétique opposée à la direction de recherche étudiée ici, mais de la compréhension génétique à la fois distincte et proche de la compréhension phénoménologique statique que Jaspers développera à la fin de l'article, ci-dessous p. 327, N.d.T.]

5. Ici les travaux de l'école de Kulpe ont été extraordinairement fertiles pour la phénoménologie. Toutes ces choses très complexes ne demandent pas à être exposées de façon détaillée ici, où on ne vise que la fixation de la direction de recherche phénoménologique. À propos de l'autoobservation, voir Elias Müller, Zur Analyse der Gedächtnistätigkeit und des Vorstellungsverlaufs, Leipzig, 1911, p. 161-176.
} 
ment, l'immersion dans la conduite, le comportement, les mouvements expressifs; deuxièmement, l'exploration avec son questionnement et les renseignements que le malade nous livre à son propos, lorsque nous l'y conduisons ; troisièmement, les descriptions de soi consignées par écrit, rarement bonnes, mais toujours très précieuses et éventuellement utilisables même sans connaissance personnelle de leur rédacteur. Dans tous ces cas, nous pratiquons la phénoménologie dans la mesure où nous sommes orientés sur le psychique et non sur les apparitions objectives qui sont au contraire seulement un point de passage, un moyen, et non un objet de recherche. Les bonnes autodescriptions sont d'une valeur toute particulière . $^{6}$

Si nous cherchons par ces moyens à approcher la vie psychique du malade, nous sommes face à un chaos durable, fluant et impénétrable, de phénomènes toujours changeants. Notre premier but doit être $d^{\prime} y$ délimiter le singulier, de le présentifier de tous côtés pour nous-mêmes comme pour les autres à des fins d'usage durable [321] et de le pourvoir d'un nom d'usage constant et identique. Les phénomènes psychopathologiques suggèrent fortement l'usage d'une telle observation phénoménologique isolée, abstraite des configurations, qui ne veut présentifier que le donné, comprendre de façon non génétique, et qui ne veut que voir, et non expliquer. De nombreux phénomenes psychiques apparaissent de façon pathologique sans condition compréhensible, surgissent, d'un point de vue psychologique, du néant, sont, d'un point de vue causal, provoqués par le processus de la maladie. Les souvenirs vivants de choses qui n'ont jamais été vécues, les pensées accompagnées de la conscience de leur exactitude sans que cette conscience soit fondée de façon compréhensible (idées délirantes), les tonalités et les affects qui apparaissent complètement spontanément, ou sans être fondés sur des vécus ou des pensées...,

\footnotetext{
${ }^{6}$. Il est incontestable que la publication de telles auto-descriptions est d'une grande valeur pour la phénoménologie. Comme ce sont les malades particulièrement instruits et intelligents qui se livrent à des auto-descriptions, les plus grands profits proviennent plutôt des médecins des institutions privées qui peuvent accéder plus facilement à telles descriptions de soi, que des médecins des institutions publiques. $\mathrm{Si}$ on sous-évalue encore souvent et considère comme insuffisant de publier un "cas", il est nécessaire de rappeler la valeur absolument extraordinaire des auto-descriptions, de les rassembler et de les exploiter plus qu'on ne l'a fait jusqu'à maintenant. Je prie les lecteurs de ces lignes, qui sont en possession de bonnes autodescriptions - $c^{\prime}$ est-à-dire de celles qui amènent intuitivement au regard les phénomènes effectivement vécus de l'âme - de les publier, ou, au cas où ils ne peuvent s'y résoudre, de me les transmettre pour une observation ou une éventuelle exploitation. Pour les lecteurs intéressés, j'indique certaines des meilleures auto-descriptions publiées jusqu'ici. Elles ne sont pas nombreuses: Schreber, Denkwürdigkeiten eines Nervenkranken, Leipzig, 1903. Th. de Quincey, Bekenntnisse eines Opiumessers, Stuttgart, 1886. Gérard de Nerval, Aurelia (en allemand), Munich, 1910, J. J. David, "Halluzinationen", Die neue Rundschau 17, 874, Wollny, Erklärungen der Tollheit von Haslam, Leipzig, 1889, Kandinsky, « Zur Lehre von den Halluzinationen », Archiv für Psychologie. 9, Kieser, Allgemeine Zeitschrift für Psychologie 10, 423, Engelken, ibid. 6, 586, Meinert, Alkoholwahnsinnig, Dresden, 1907.
} 
sont des manifestations fréquentes. Elles sont l'objet de la recherche phénoménologique qui constate et présentifie la manière dont elles sont véritablement. Trois groupes de phénomènes sont ainsi à dégager. Les uns sont connus de nous tous dans le vécu personnel propre. Ils ont la même constitution que les processus psychiques analogues qui sont conditionnés de façon normalement compréhensible. Ce n'est que par leur genèse que se distinguent les phénomènes pathologiques, par ailleurs parfaitement identiques à ceux de l'homme sain, par exemple les falsifications de souvenirs. Nous devons saisir les deuxièmes comme les intensifications, les ralentissements ou les mélanges des phénomènes vécus en personne, par exemple le saisissement béat de la psychose aiguë, les pseudos hallucinations, les excitations pulsionnelles perverses. On ne peut répondre définitivement à la question de savoir jusqu'où va notre présentifier compréhensif des vécus des malades lorsqu'il est privé du fondement que constituent nos propres vécus conscients qui ont des directions semblables à ceux des malades. Il semble parfois que notre compréhension va loin au-delà de la possibilité de notre seul vécu propre. Le troisième groupe de phénomènes pathologiques se distingue du dernier groupe par sa totale inaccessibilité à un présentifier compréhensif. Nous nous en approchons seulement par des analogies et des images. Et nous les remarquons dans les cas particuliers non par une compréhension positive, mais par le heurt avec cet incompréhensible dont le cours de notre compréhension fait l'expérience. À ce domaine appartiennent par exemple les pensées "fabriquées ", les tonalités "fabriquées ", etc., que de nombreux malades décrivent avec assurance comme des vécus, mais que nous n'identifions néanmoins jamais que par ce type d'expressions [ "fabriquées »] et par des constats relatifs à ce dont il ne s'agit pas dans ces phénomènes. Certains malades, qui dans leur psychose ont encore une conscience de la vie psychique normale, reconnaissent eux-mêmes l'impossibilité de décrire leurs vécus dans la langue usuelle. Un malade expliquait « qu'il s'agit ici de choses qui ne se laissent en général jamais exprimer dans la langue humaine... pour devenir en quelque sorte compréhensible, il me faut recourir à des images et des paraboles qui ne sont peut-être qu'approximatives ; car le seul chemin, c'est la comparaison avec les états de faits connus de l'expérience humaine ... [autre passage] à cela s'ajoute le fait qu'il $s^{\prime}$ agit ici en grande partie de visions dont j'ai certes les images dans la tête, mais dont la description en mots est singulièrement difficile, en partie vraiment impossible ». Certains des neologismes des malades - mais pas tous - reposent sur de telles dénominations de vécus entièrement propres. [322] Un malade cherchait à décrire plus précisément une sensation dans la hanche en répondant à la question de savoir s'il s'agissait de spasmes : "Non, ce ne sont pas des spasmes (Zuckungen), ce sont des zoupements (Zoppungen)». 
Dès ses commencements, la psychiatrie a déjà travaillé dans cette direction d'une délimitation et d'une dénomination des formes singulières de vécus, et elle n'aurait pas pu faire un seul pas sans une telle fixation phénoménologique. Ainsi furent, entre autres, décrits les idées délirantes, les illusions des sens, les affects dépressifs et expansifs. Tout ceci restera le fondement de constatations phénoménologiques plus poussées. Mais ces descriptions doivent souvent être purifiées du fatras des réflexions théoriques relatives aux fondements corporels et aux configurations psychiques construites. De nombreux commencements phénoménologiques ont été étouffés par de tels efforts théoriques. Nous ne nous satisferons plus de quelques concepts économiques, mais nous nous dévouerons sans présupposé aux phénomènes, et là où nous en verrons un, nous chercherons à nous le présentifier pleinement - sans présomption préalable de ce qu'est ce phénomène et sans appel à nos connaissances psychologiques. L'habituelle partition des symptômes d'égarement en illusions de sens et en idées délirantes est certes utile en tant que bref mot d'ordre, mais ces désignations recouvrent une foule considérable de phénomènes très differents. Quelques exemples devraient permettre d'intuitionner ce qui doit être fixé pour les phénomènes. a) Kandinsky décrit les pseudos hallucinations comme un certain genre de représentations pathologiques. Elles se distinguent des représentations normales par une grande détermination sensible, une clarté, un détail, par leur apparition indépendamment de la volonté et contre elle, ainsi que par le vécu de la passivité et de la réceptivité. Mais elles se distinguent aussi des perceptions trompeuses comme des perceptions normales par une apparition non dans l'espace extérieur en même temps que les perceptions, mais dans l'espace intérieur, dans lequel nous voyons aussi des représentations devant nous. On a considéré ces pseudos hallucinations dans le cadre d'une pensée théorique. Il s'agit cependant d'un problème exclusivement phénoménologique et descriptif. On peut présentifier les cas décrits d'une manière peut-être phénoménologiquement plus évidente, autrement que ne le fait Kandinsky. Pour ce faire, on peut en appeler à d'autres cas (auto-descriptions, résultats d'explorations), mais on ne peut réfuter Kandinsky que par de telles présentifications intuitives, jamais par des réflexions théoriques. La conscience de l'autonomie de la tâche phénoménologique garantit des critiques qui se méprennent sur elle et sont en cela stériles. b) Il n'est pas rare que les malades vivent un phénomène dans lequel ils prennent conscience de façon pressante que quelqu'un est derrière eux ou au-dessus d'eux. Ce quelqu'un se tourne avec eux, lorsqu'ils regardent alentour. Ils le « sentent », il y a «effectivement » quelqu'un la. Mais ils ne ressentent aucun contact, ils n'éprouvent rien du tout, ils ne peuvent non plus en avoir la vision. Certains malades jugent malgré tout qu'il n'y a là personne, d'autres sont 
convaincus de l'existence de ce quelqu'un dont ils ressentent vivement la présence. Il ne s'agit manifestement pas ici d'illusions des sens, dans la mesure où manque l'élément sensible, il ne s'agit pas non plus d'idées délirantes dans la mesure où un vécu est présent, qui est seulement réélaboré dans le jugement, correctement ou de façon délirante $^{7}$. [323] c) Un troisième exemple encore, tiré de la sphère des sentiments, doit permettre d'intuitionner que, par la pure immersion dans des phénomènes singuliers sans systeme et sans théorie, on en vient à une présentification et à une détermination de phénomènes d'abord simplement juxtaposés. On parle de sentiments extatiques. En ceux-ci, on peut différencier sinon différents phénomènes du moins différentes nuances - cela dépend naturellement ici de l'exactitude de l'exemple singulier dont on se sert. On trouve premièrement une ascension, une émotion, un saisissement au-delà du possible, deuxièmement une béatitude intérieure profonde qui produit çà et là une représentation réconfortante, troisiemement un sentiment d'élévation et de grâce, de salut et de grande signification. De telles distinctions, brièvement esquissées, ont besoin, si elles doivent avoir de la valeur, d'être élaborées par la phénoménologie, respectueuse d'une position correcte et $d$ 'une fixation phénoménologique.

Nous connaissons maintenant les moyens de la phénoménologie psychopathologique (mouvements d'expression, exploration, autodescription) et les voies à suivre pour la présentification propre (genèses, conditions, constellations de l'apparition des phénomènes, contenus de ces mêmes phénomènes, les éléments déjà connus présents en eux, les renvois symboliques, etc.), et nous savons qu'il ne reste plus que l'appel fait à chacun à se présentifier le phénomene en prenant en considération ce qui vient d'être dit. Dans un travail phénoménologique apparaîtront alors des cas singuliers qui permettront de parvenir à des descriptions générales, d'établir des dénominations. Le fait que la phénoménologie nomme simplement ce qui est donné immédiatement n'est pas une objection, mais seulement une constatation. C'est seulement qu'il est toujours difficile d'acquérir une compréhension plus générale dans le cas singulier et une relative complétude de la délimitation phénoménologique. Il faut considérer ici que le vécu d'un malade particulier est toujours infiniment divers, que la phénoménologie n'en tire cependant que quelque chose de général, qui est tout aussi général dans le vécu d'un autre cas, et auquel nous donnons pour cette raison le même nom, alors que cette infinité de l'individuel change toujours. Il en résulte que la phénoménologie, d'un côté abstrait quelque chose de l'infinité des éléments changeants, et d'un autre côté est tournée vers quelque chose qui n'est absolument

\footnotetext{
7. Je décrirai bientôt ce genre de phénomènes ailleurs en m'appuyant sur des cas de " consciences [auxquelles un phénomène] est concrètement donné ».
} 
pas abstrait, mais complètement intuitif. C'est seulement dans la mesure où quelque chose demande à être effectivement donné immédiatement, c'est-à-dire est intuitif, que cette chose est un objet pour la phénoménologie.

Admettons que, par la délimitation descriptive phénoménologique, une série de phénomènes puisse être généralement présentifiée et rendue consciente. Maintenant, nous sommes manifestement pour la deuxième fois face à un chaos d'innombrables phénomènes cette fois doués d'une dénomination, mais qui ne satisfont encore nullement notre besoin scientifique. La mise en ordre doit permettre de délimiter des phénomènes singuliers pour parvenir méthodiquement à la conscience de la multiplicité du psychique et, au niveau atteint par la recherche, à une visibilité des phénomènes. [324] On peut ordonner les phénomènes très différemment selon le but que l'on a chaque fois. On peut ordonner, par exemple, selon la genèse, selon les éventuelles conditions corporelles, selon les contenus, selon la signification que les phénomènes ont d'un point de vue quelconque (ainsi les phénomènes logiques, éthiques et esthétiques du psychique). On donnera à chacun de ces principes d'ordre son droit en son lieu. Ils sont peu satisfaisants pour la phénoménologie elle-même. Nous cherchons ici plutôt un ordre qui juxtapose les phénomènes selon leur parenté phênoménologique, tout comme les couleurs en nombre infini sont rendues visibles dans leur ensemble de manière phénoménologiquement satisfaisante dans le cercle ou la sphère des couleurs. En l'état actuel de la phénoménologie, il s'avère maintenant, qu'il y a des groupes de manifestations entre lesquels il $n^{\prime} y$ a aucune parenté remarquable : les impressions sensibles et les pensées, les perceptions trompeuses et les jugements délirants, sont séparés par un abîme; ce ne sont pas des phénomènes liés par des transitions. De tels phénomènes sans parenté se laissent seulement juxtaposer et ne peuvent être ordonnés plus avant. On ne peut globalement pas voir jusqu'où des séparations se laissent finalement réduire à une ou à quelques différences fondamentales à l'intérieur du psychique.

Face aux phénomènes complètement separés les uns des autres se tiennent de l'autre côté des groupes visiblement ordonnés, des manifestations apparentées les unes aux autres. Entre celles-ci, il y a des transitions comme entre les couleurs. Les pseudos hallucinations sont un exemple ${ }^{8}$ de ce genre de phénomènes apparentés en une vue d'ensemble. Il s'avère lors d'une observation plus précise de cas particuliers qu'il y a des transitions entre les représentations normales et les pseudos hallucinations complètement fabriquées (qui ne sont jamais données en chair et en os et demeurent toujours dans l'inté-

\footnotetext{
${ }^{8}$. Il ne me semble pas important $i c i$ que l'exemple soit correctement choisi. Cela doit seulement servir l'intuition du but.
} 
riorité de l'espace de représentation). Pour rendre ces transitions pleinement visibles, il suffit de considérer quatre oppositions principales entre lesquelles ces phénomènes peuvent osciller dans une série de transitions. Si nous décrivons quelque chose comme un lieu en chacune de ces séries, nous avons suffisamment caractérisé le phénomène particulier qui se tient entre représentation et pseudo hallucination. Ces quatre oppositions sont :

\begin{tabular}{|l|l|}
\hline \multicolumn{1}{|c|}{ Pseudos hallucinations fabriquées } & \multicolumn{1}{|c|}{ Représentations normales } \\
\hline $\begin{array}{l}\text { 1. Elles ont un tracé déterminé, se } \\
\text { tiennent entièrement devant nous } \\
\text { avec tous les détails. }\end{array}$ & $\begin{array}{l}\text { Elles ont un tracé indéterminé, ne se } \\
\text { tiennent pas complètement devant } \\
\text { nous, mais seulement dans certains } \\
\text { détails. }\end{array}$ \\
\hline $\begin{array}{l}\text { 2. Rapportées aux éléments parti- } \\
\text { culiers de l'impression, elles ont } \\
\text { une totale adéquation aux percep- } \\
\text { tions correspondantes. }\end{array}$ & $\begin{array}{l}\text { Elles n'ont cette adéquation que } \\
\text { dans peu d'éléments de l'impres- } \\
\text { sion, ou n'en ont pas du tout. Par } \\
\text { exemple, dans une représentation } \\
\text { visuelle, tout est gris. }\end{array}$ \\
\hline $\begin{array}{l}\text { 3. Elles sont constantes et peuvent } \\
\text { facilement être maintenues de la } \\
\text { même manière. }\end{array}$ & $\begin{array}{l}\text { Elles s'écoulent, se dispersent, et } \\
\text { doivent être toujours produites à } \\
\text { nouveau. }\end{array}$ \\
\hline $\begin{array}{l}\text { 4. Elles sont indépendantes de la } \\
\text { volonté et ne peuvent être appe- } \\
\text { lées à volonté ni changées. Elles se } \\
\text { donnent dans un sentiment de } \\
\text { passivité et de réceptivité. }\end{array}$ & $\begin{array}{l}\text { Elles sont dépendantes de la vo- } \\
\text { lonté, peuvent être appelées à } \\
\text { volonté et peuvent être changées. } \\
\text { Elles sont produites avec le senti- } \\
\text { ment de l'activité. }\end{array}$ \\
\hline
\end{tabular}

[325] Cet exemple, dans lequel nous n'entrons pas plus avant, montre comment il est possible de regrouper de manière purement phénoménologique des phénomènes apparentés dans la mesure où seuls les côtés effectivement vécus de ces phénomènes constituent les critères de leur répartition, alors que les pensées additionnelles, le théorique, demeurent encore très lointains. Pour le dire brièvement, l'importance de la distinction entre les transitions phénoménologiques et les abîmes phénoménologiques se déduit de ce qui a été dit antérieurement. Les premières permettent d'ordonner phénoménologiquement, les derniers permettent seulement des oppositions et des énumérations. Il est alors naturel que l'on se résolve toujours difficilement, et alors seulement par présentification intuitive, à la reconnaissance d'un nouveau groupe phénoménologique de phénomènes séparés par un abîme des autres phénomènes rencontrés jusqu'ici. Malgré tout, à l'heure actuelle où beaucoup veulent ramener le plus possible 
tout le psychique à un nombre réduit de qualités simples, il est préférable $\mathrm{d}$ 'admettre trop de phénomènes - que l'on ordonnera bientôt de toute façon - que de sombrer dans la platitude de systèmes psychologiques construits à partir de quelques éléments.

Alors que l'idéal de la phénoménologie est une infinité entièrement visible de qualités psychiques irréductibles, il y a, opposé à lui, un autre idéal, celui du plus petit nombre possible d'éléments ultimes, comme en chimie par exemple. Tous les phénomènes complexes de l'âme doivent être déduits de leur combinaison, et tous doivent être suffisamment présentés par l'analyse en de tels éléments. Il résulte finalement qu'une telle conception ne peut pas considérer comme insensé de partir d'un unique atome singulier de l'âme à partir duquel tout le psychique se construit en diverses configurations. Cet idéal orienté sur les sciences de la nature a certainement un sens pour la genèse des qualités psychiques. On peut souhaiter éclairer génétiquement les autres qualités psychiques de la même façon que les couleurs infiniment nombreuses peuvent être simplement ramenées à quelques oscillations quantitatives, et il est peut-être possible de les ordonner autrement de ce point de vue. Mais pour la phénoménologie elle-même, cette exigence semble insensée. L'analyse phénoménologique a pour but de prendre conscience des phénomènes psychiques en les circonscrivant clairement. Dans ce cadre elle procède alors entre autres par l'exposition de qualités psychiques qui se produisent en tant que parties dans le phénomène visé. Cette décomposition de formations complexes en de telles parties, qui n'est qu'un chemin, est tenue pour la seule analyse possible par les tenants de la conception pour laquelle seule la genèse importe. Pour une telle conception, la perception, par exemple, serait éclairée par la décomposition en éléments d'impression, intuition spatiale, et d'acte intentionnel, alors que la vraie phénoménologie n'arrive à une caractérisation de la perception en tant que qualité psychique irréductible que par la comparaison avec la représentation qui est construite des mêmes éléments, avec, par exemple, le jugement. Si la conception de «l'analyse en éléments ultimes » réussit, autant que "l'analyse en tant que délimitation des qualités ultimes ", à passer pour libre de tout point de vue génétique et pour phénoménologique, elle retombe cependant en chaque occasion dans la méprise de l'observation génétique : pour elle, les formations complexes naissent des éléments en composition. [326] Contre ces intuitions, la phénoménologie n'a pas pour idéal le plus petit nombre possible d'éléments ultimes. Au contraire, elle ne veut pas borner l'infinité des phénomènes psychiques, mais dans la mesure du possible - c'est bien sûr une tâche infinie - les rendre entièrement visibles, se les rendre clairement conscients et reconnaissables dans leur singularité.

Même si c'est seulement à grands traits, nous avons exposé les buts et la méthode de la phénoménologie, qui a certes toujours été 
pratiquée, mais n'en vint jamais vraiment à un développement sans obstacles. Puisque son mélange avec d'autres tâches de recherche fut toujours son plus grand dommage, nous voulons encore énumérer ce que la phénoménologie ne veut pas, et ce avec quoi il n'est pas permis de la confondre.

La phénoménologie a seulement affaire à ce qui est effectivement vécu, seulement à l'intuitif, non à des choses quelles qu'elles soient, qui sont pensées au fondement du psychique, qui sont construites théoriquement. En chacune de ses découvertes, elle doit se demander : ceci est-il effectivement vécu ? Ceci est-il aussi effectivement donné dans la conscience? Ce qu'elle établit tient sa certitude du fait qu'il s'avère toujours possible de représentifier l'effectivité psychique, et ne peut être réfuté que dans la mesure où les éléments jusqu'ici faussement présentifiés le sont maintenant correctement, et non parce qu'on peut mettre en évidence l'incorrection ou l'impossibilité de propositions théoriques quelconques. La phénoménologie ne peut rien acquérir par la théorie, mais a au contraire tout à perdre. L'exactitude de la présentification singulière n'est pas à contrôler à partir de critères généraux. Elle doit toujours trouver sa mesure en elle-même.

La phénoménologie a affaire à ce qui est effectivement vécu. Elle voit le psychique "de l'intérieur » dans une présentification immédiate. Elle n'a ainsi pas affaire aux investigations relatives aux phénomènes d'extériorisation, aux phénomènes moteurs, aux mouvements expressifs en tant que tels, aux opérations objectives. Dans quelle mesure les mouvements expressifs et les auto-descriptions ne sont pas les objets mais les moyens de la phénoménologie, cela a été montré plus haut.

La phénoménologie $n^{\prime}$ a en outre pas affaire à la genèse des phénomènes psychiques. Elle n'est que la condition préalable pour de telles recherches génétiques, mais elle-même les laisse complètement de côté et ne peut être réfutée ni requise par de telles recherches. La recherche relative à la naissance des couleurs, des perceptions, etc., est étrangère à la phénoménologie. De telles recherches génétiques ont de fait été moins dangereuses pour elle que les «mythologies cérébrales » qui interprètent la phénoménologie et la remplacent par des constructions de processus cérébraux physiologiques et pathologiques. Wernicke, qui a fait des constats phénomenologiques significatifs, les a déformés en les interprétant ainsi en termes, par exemple, de fibres associatives, de séjonction... ${ }^{9}$ Ces constructions ne permettent habituellement pas à la phénoménologie de se déployer pleinement. Poussées par la nécessité, elles doivent certes pratiquer la phénoménologie à leur commencement, mais lorsqu'elles sont arri-

${ }^{9}$. La « séjonction » ou disjonction est un concept forgé par Wernicke pour rendre compte de la dementia sejunctiva ou schizophrénie (Note de l'éditeur). 
vées à leur théorie, elles se sentent sur un sol plus sûr et, dans une méconnaissance de leur propre source, elles trouvent le phénoménologique trop «subjectif ».

[327] Enfin, l'observation phénoménologique est aussi à séparer de la compréhension génétique des processus psychiques, de cette compréhension spécifique qui n'est applicable qu'au psychique, pour laquelle le psychique «surgit » avec évidence du psychique, et pour laquelle il va de soi que l'agressé devient colérique, que l'amant trompé devient jaloux. On parle de "compréhension » aussi bien dans la présentification phénoménologique que dans la saisie de ce surgissement de l'un à partir de l'autre. Pour éviter la confusion, nous nommons la compréhension phénoménologique des états psychiques, qui ne saisit que les données, les vécus, les modes de conscience, et qui est le fondement de sa délimitation et de sa caractérisation, la compréhension statique. La compréhension des configurations des vécus psychiques, $\mathrm{du}$ surgissement du psychique depuis le psychique, nous le nommons la compréhension génétique. La phénoménologie n'a pas affaire à cette compréhension génétique, elle est plutôt traitée séparément de cette compréhension, elle a pour objet les continuités régulières du psychique qui sont effectivement vécues et forment une unité phénoménologique spécifique. Cette continuité phénoménologique n'a rien à voir avec un surgissement de l'un à partir de l'autre. Nous bornons la phénoménologie au compréhensible statique.

Il va de soi que lorsque nous considérons la psychopathologie en son ensemble, notre véritable intérêt se focalise sur le compréhensible génétique, les relations de dépendance extra-conscientes, le constat de causes corporelles des processus psychiques, en un mot sur les configurations. La phénoménologie nous apprend seulement à connaître les formes dans lesquelles tout vécu, toute effectivité psychique arrive, elle ne nous enseigne pas à connaître les contenus du vécu singulier, ni les fondements extra-conscients sur lesquels flotte le psychique comme la mince surface de l'écume flotte sur la mer. Il sera toujours plus excitant de pousser dans les profondeurs de l'extraconscient à travers la connaissance des configurations, que de faire de simples constatations phénoménologiques dont l'élucidation exacte est cependant la condition préalable de toute recherche postérieure. C'est seulement dans les formes découvertes phénoménologiquement que se déroule la vie psychique effective qui nous est immediatement accessible et qui, pour être conçue, appelle en fin de compte l'investigation des seules configurations extra-conscientes.

Pour conclure, indiquons quelques tâches particulières de la phénoménologie. Il n'y a en général aucun domaine présent de la phénoménologie psychopathologique qui soit clos. Même là où un phénomène est intuitivement clair, comme dans certaines perceptions trompeuses, la bonne casuistique, qui peut servir en tant que donnée 
empirique, est si rare que les cas soigneusement décrits ont toujours une grande valeur. Il y a encore beaucoup à faire au sujet des types de perceptions trompeuses qui restent à examiner au sens indique cidessus. Il suffit de penser simultanément à la question des illusions visuelles dans l'espace objectif et aux perceptions réelles. La phénoménologie des vécus délirants a à peine été abordée. Ce qui existe à ce sujet se trouve dans les travaux sur les sentiments en tant que premiers symptômes de la paranoïa. La phénoménologie des sentiments pathologiques est incroyablement pauvre. Le meilleur se trouve dans les travaux remarquables de Janet [328] dans lesquels on accorde cependant peu de valeur à la délimitation rigoureuse et à la mise en ordre ${ }^{10}$. La conscience de la personnalité est travaillée systématiquement par Oesterreich. Pour ces problèmes, les descriptions phénoménologiques de psychiatres qui disposent du matériau et les auto-descriptions plus détaillées que celles produites jusqu'ici auraient une grande valeur.

En histologie, il est nécessaire d'examiner chaque fil et chaque granule lors de l'étude des couches cérébrales. De manière tout à fait analogue, la phénoménologie émet ses exigences: on doit rendre compte de chaque phénomène psychique, de chaque vécu, qui apparaît lors de l'exploration des malades et de leur auto-description. On ne doit en aucun cas se satisfaire de l'impression générale et de quelques détails ad hoc, mais savoir à propos de chaque singularité comment elle demande à être saisie et jugée. Si l'on procède quelque temps ainsi, d'un côté on sera moins étonné de ce que l'on aura fréquemment vu et de ce dont celui qui ne travaille qu'avec une impression globale n'est pas devenu conscient, et dont, conformément à l'orientation toujours fugace de son impressionnabilité, il est toujours prêt à s'étonner en le considérant comme absolument nouveau ; mais d'un autre côté, si on prête attention à ce qui nous est effectivement inconnu, on est à juste titre étonné. Il n'y a pas de danger que cet étonnement cesse.

Il va de soi que beaucoup de psychiatres procèdent déjà ainsi et ressentiraient à juste titre comme une présomption le fait que cela leur soit de nouveau demandé. Mais l'attitude phénoménologique n'est pas si répandue que l'on n'ait pas besoin de l'exiger toujours de nouveau. On est en droit d'espérer que cette attitude contribue décisivement à enrichir la connaissance de ce que le malade vit effectivement.

[Traduction Simon Calenge]

\footnotetext{
${ }^{10}$ L'extension de la phénoménologie des sentiments devra prendre en compte avant tout les travaux de Geiger : "Das Bewußtsein der Gefühle», Münchner Philosophische Abhandlung, Th. Lipps zu seinem 60. Geburtstag gewidmet, et : «Zum Problem der Stimmungseinfühlung », in Zeitschrift für Ästhetik 6, 1, 1911.
} 